\title{
Angiocrine Hepatocyte Growth Factor Signaling Controls Physiological Organ and Body Size and Dynamic Hepatocyte Proliferation to Prevent Liver Damage during Regeneration

Xue-jun Zhang, ${ }^{\dagger \dagger}$ Victor Olsavszky, ${ }^{\ddagger}$ Yuhan Yin, ${ }^{\star}$ Baocai Wang, ${ }^{*}$ Thomas Engleitner, ${ }^{\S}$ Rupert Öllinger, ${ }^{\S}$ Kai Schledzewski, Philipp-Sebastian Koch, ${ }^{\ddagger}$ Roland Rad, ${ }^{\S}$ Roland M. Schmid, ${ }^{\top}$ Helmut Friess, ${ }^{*}$ Sergij Goerdt, ${ }^{\ddagger}$ Norbert Hüser, ${ }^{*}$ Cyrill Géraud, ${ }^{\ddagger} \|_{* *}$ Guido von Figura, " and Daniel Hartmann*

From the Department of Surgery, * Translatum Cancer Center, and Department of Medicine II, ${ }^{\S}$ Institute of Molecular Oncology and Functional Genomics, and II: Medical Clinic and Policlinic, "Technical University of Munich, School of Medicine, Klinikum rechts der Isar, Munich, Germany; the Department of Orthopedic Surgery, ${ }^{\dagger}$ Zhongda Hospital, School of Medicine, Southeast University, Nanjing, China; the Department of Dermatology, Venereology, and Allergology, ${ }^{\ddagger}$ University Medical Center and Medical Faculty Mannheim, Heidelberg University, Center of Excellence in Dermatology, Mannheim, Germany; and the Section of Clinical and Molecular Dermatology" and the European Center for Angioscience, ** Medical Faculty Mannheim, Heidelberg University, Mannheim, Germany

Accepted for publication

October 22, 2019.

Address correspondence to Cyrill Géraud, M.D., Department of Dermatology, Venereology, and Allergology, University Medical Center Mannheim, Theodor-Kutzer-Ufer 1-3, Mannheim 68167, Germany; or Daniel Hartmann, M.D., M.Sc., Ph.D., Technical University of Munich, School of Medicine, Klinikum rechts der Isar, Department of Surgery, Munich 81675, Germany. Email: cyrill.geraud@umm.de or daniel. hartmann@tum.de.

\begin{abstract}
Liver sinusoidal endothelial cells (LSECS) control organ functions, metabolism, and development through the secretion of angiokines. LSECs express hepatocyte growth factor (Hgf), which is involved in prenatal development, metabolic homeostasis, and liver regeneration. This study aimed to elucidate the precise contribution of LSEC-derived $\mathrm{Hgf}$ in physiological homeostasis and liver regeneration. Stab2-iCre $e^{t g / w t} ; \mathrm{Hgf}^{f / f l}\left(\mathrm{Hgf}^{\Delta \mathrm{LSEC}}\right)$ mice were generated to abrogate $\mathrm{Hgf}$ expression selectively in LSECs from early fetal development onwards, to study global development, metabolic and endothelial zonation, and organ functions as well as liver regeneration in response to $70 \%$ partial hepatectomy $(\mathrm{PH})$. Although zonation and liver/body weight ratios were not altered, total body weight and total liver weight were reduced in $\mathrm{Hg}^{\mathrm{f}^{\mathrm{LSEC}}}$. Necrotic organ damage was more marked in $\mathrm{Hgf}^{\mathrm{ALSEC}}$ mice, and regeneration was delayed 72 hours after $\mathrm{PH}$. This was associated with decreased hepatocyte proliferation at 48 hours after PH. Molecularly, $\mathrm{Hgf}^{\Delta L S E C}$ mice showed down-regulation of $\mathrm{Hgf} / \mathrm{c}-$ Met signaling and decreased expression of Deptor in hepatocytes. In vitro knockdown of Deptor was associated with decreased proliferation. Therefore, angiocrine $\mathrm{Hgf}$ controls hepatocyte proliferation and susceptibility to necrosis after partial hepatectomy via the $\mathrm{Hgf} / \mathrm{c}-$ Met axis involving Deptor to prevent excessive organ damage. (Am J Pathol 2020, 190: 358-371; https://doi.org/10.1016/j.ajpath.2019.10.009)
\end{abstract}

Hepatocyte growth factor (Hgf) was first isolated from rat serum after partial hepatectomy $(\mathrm{PH})$, thereby associating its function with liver regeneration. ${ }^{1,2}$ Nowadays, it is known that Hgf can be secreted by different cell types of mesenchymal origin in various organs, such as the lung, liver, brain, thyroid, and salivary gland. ${ }^{3,4}$ After binding to its tyrosine kinase receptor, c-Met, dimerization and phosphorylation of the C-terminal receptor domain ensues, leading to interactions with multiple signal transducers, such as Stat3, growth factor receptor-bound protein 2 (Grb2),
Supported by the Wilhelm Sander-Stiftung project 2016.033.1 (D.H. and G.v.F.); and the Deutsche Forschungsgemeinschaft (German Research Foundation) projects 259332240/RTG 2099 (C.G., S.G., and P.-S.K.), 5454871/SFB TR23 (C.G. and S.G.), and 394046768/SFB 1366 (C.G., S.G., and P.-S.K.). X.-j.Z. and B.W. were supported by the China Scholarship Council. Work in G.v.F.'s laboratory was supported by funding from the Deutsche Forschungsgemeinschaft Emmy Noether Program FI1719/2-1.

X.-j.Z. and V.O. contributed equally to this work.

C.G., G.v.F., and D.H. contributed equally as senior authors.

Disclosures: None declared. 
Src homology 2 domain containing (Shc), or phospholipase $\mathrm{C} \gamma$ (Plcã). ${ }^{5,6}$ Hgf signaling induces diverse biological activities, including morphogenesis, mitogenesis, and cytoprotection. ${ }^{7}$ Constitutive and global knockout of $\mathrm{Hgf}$ and $\mathrm{c}$ Met in mice are lethal during development between embryonic day (E) 12.5 and E16.5, with Hgf knockout embryos showing a severely reduced liver size. ${ }^{8,9}$ The Hgf/cMet axis is also involved in the physiological homeostasis and regeneration of many extrahepatic organs, like the heart, kidney, lung, gut, skin, and others. ${ }^{10}$

In the adult liver, Hgf is expressed by Kupffer cells, stellate cells, and liver sinusoidal endothelial cells (LSECs). ${ }^{11-13}$ Moreover, after liver damage, $H g f$ gene expression shows up-regulation in endothelial cells. ${ }^{11}$ Previous studies have shown that LSECs secrete several hepatotrophic proteins, such as Hgf, Bmp2, Wnt9b, and Wnt2, to stimulate liver regeneration and control metabolic functions. ${ }^{14-17}$ Such endothelial cell-derived paracrine acting factors are also known as angiocrine factors or angiokines. ${ }^{18}$ Angiocrine Bmp2 and Wnt signaling pathways were shown to control whole body iron metabolism and metabolic liver zonation, respectively, under steady state. ${ }^{16,17} \mathrm{Hgf}$ and Wnt2 induced hepatocyte proliferation after $\mathrm{PH} .{ }^{14}$ Besides these angiokines, endothelial angiopoietin-2 (Ang2), transforming growth factor- $\beta 1$, vascular endothelial growth factor receptor 2, fibroblast growth factor receptor 1, CXC chemokine receptor 4 (Cxcr4), and Cxcr7 are involved in the orchestrated sequence of the different phases of regeneration and control the balance between physiological regeneration and pathologic fibrosis after tissue damage. ${ }^{13,19}$ Besides its function in liver regeneration, the Hgf/c-Met axis is involved in melanoma metastasis to the liver and constitutive overexpression of hepatic Hgf promotes tumor development. ${ }^{20,21}$ Circulating Hgf levels are involved in the regulation of insulin resistance and obesity on high-fat diet. In this regard, transgenic overexpression of Hgf correlates with decreased body weight. ${ }^{22}$

$\mathrm{PH}$ is the most commonly studied experimental model in liver regeneration. As early as 2 hours after $\mathrm{PH}$, Hgf protein concentration in the plasma increases $>10$-fold; and mRNA expression in the liver peaks at 12 hours after $\mathrm{PH}^{4,23}$ In addition, $H g f$ expression does not only increase in the liver, but also in lungs, ${ }^{24}$ spleen, and kidneys. ${ }^{25}$ As constitutive knockout of $\mathrm{Hgf}$ and $c$-Met in mice is lethal during development, inducible knockout mice have been facilitated to study their functions during regeneration. Inducible hepatocyte-specific $c$-Met knockout in adult mice does not compromise physiological liver function and structure. However, these mice die 48 hours after PH and exhibit liver necrosis and diffuse macrovesicular or microvesicular steatosis, indicating that c-Met activation is required for liver regeneration but not for physiological maintenance of hepatic functions in adult mice. ${ }^{26}$

To study endothelium-derived $\mathrm{Hgf}$ in adult mice, $\mathrm{PH}$ has been performed on mice with an endothelial-specific tamoxifen-induced vascular endothelial-cadherin-Cre-mediated deletion of $\mathrm{Hgf}$. After $\mathrm{PH}$, these mice showed impaired regeneration, indicating that angiocrine $\mathrm{Hgf}$ cannot be compensated by other Hgf-expressing cells. ${ }^{27}$ Although it was not specifically reported in this publication, these mice appeared to have normal physiological liver functions when not being challenged with pathologic stimuli. Stab2-Cre mice ${ }^{16,28}$ that exhibit expression of the Cre-recombinase in sinusoidal endothelial cells were used to generate an $H g f$ knockout in LSECs $\left(\mathrm{Hgf}^{\triangle \mathrm{LSEC}}\right)$ that is active from E9.5 onwards and thereby allows the comprehensive analysis of angiocrine $\mathrm{Hgf}$ signaling during liver development as well as physiological homeostasis and regeneration during adulthood.

\section{Materials and Methods}

\section{Animals}

All animals were housed under specific-pathogen-free conditions. Hgf loss of function in LSECs (Stab2-iCre $e^{t g / w t}$; $H g f^{f / f l}=\mathrm{Hgf}^{\Delta \mathrm{LSEC}}$ ) was achieved by crossing Stab2-iCre ${ }^{\text {tg/wt; }}$; $H g f^{f / w t}$ with $H g f^{f / f t}$ mice. $^{29}$ Animal experiments were performed in accordance with Federal Animal Regulations and were institutionally approved by the District Government of Upper Bavaria and the district government Karlsruhe and performed under institutional guidelines (ROB-55.22532.Vet_02-18-64).

\section{Partial Hepatectomy}

$\mathrm{PH}$ of $70 \%$ was performed by removal of the left lateral lobe and the median lobe, following published methods. ${ }^{30} \mathrm{Op}$ erations were performed under general anesthesia with inhaled isoflurane between 8 and $12 \mathrm{Am}$ in the morning. A 3$\mathrm{cm}$-long midline abdominal skin and muscle incision was used in PH. Male mice at the age of 8 to 12 weeks, kept on a 12-hour day/night cycle with free access to food and water, were used in all experiments. After $\mathrm{PH}$, all experimental mice were regularly examined to recognize pain, distress, and discomfort. The following parameters were applied: no reaction, aggressiveness, or expressions of pain during handling, pain when walking, permanent chewing attitude, self-isolation, abnormal posture, paralysis, wound dehiscence, and $>20 \%$ weight loss. Once a mouse reached the predefined limit of distress, the mice were immediately euthanized. Mice were given i.p. injections of $1 \mathrm{mg}$ bromodeoxyuridine (BrdU; BD Biosciences, Heidelberg, Germany) 2 hours before euthanasia at different time points $(0$, $12,24,48,72,96$, and 168 hours) after surgery. Necropsy was performed immediately after euthanasia. Removed liver lobes were immediately weighed, fixed by $4 \%$ paraformaldehyde or flash frozen in liquid nitrogen, and stored at $-80^{\circ} \mathrm{C}$ for subsequent genomic and proteomic analysis.

\section{Immunohistochemistry and Immunofluorescence}

Liver tissue samples fixed by $4 \%$ paraformaldehyde at room temperature for 48 to 72 hours were subsequently 
Table 1 Quantitative Real-Time RT-PCR Primer Sequence Information

\begin{tabular}{lll}
\hline Gene & Forward sequence & Reverse sequence \\
\hline Hgf & $5^{\prime}$-GGTTTGGCCATGAATTTGACCT-3' & $5^{\prime}$-GGCAAAAAGCTGTGTTCATGGG-3' \\
Gapdh & $5^{\prime}$-AGGTCGGTGTGAACGGATTTG-3' & $5^{\prime}-$ TGTAGACCATGTAGTTGAGGTCA-3' \\
\hline
\end{tabular}

transferred into phosphate-buffered saline, dehydrated in a graded alcohol series, and embedded in paraffin. Paraffinembedded liver tissues were divided into sections $(3.5 \mu \mathrm{m}$ thick). Five random areas of at least five samples per group were used for blinded quantification (X.-j.Z.) of BrdU- and Ki-67-positive hepatocytes. At least five random $\times 200$ fields in more than five different sections were examined per group per time point. Hepatocyte size was determined by the average of the number of hepatocytes per field. Liver necrosis was quantified by using the percentage of necrotic area per section. For immunofluorescence, antigen retrieval of tissue sections was performed with epitope retrieval solution (Zytomed Systems, Berlin, Germany) at $\mathrm{pH} 6, \mathrm{pH} 8$, or $\mathrm{pH}$ 9. At first, antibody was incubated overnight at $4{ }^{\circ} \mathrm{C}$, and secondary antibody was applied for 1 hour at room temperature after three washing steps with phosphatebuffered saline. Sections were mounted with Dako fluorescent mounting medium (Dako, Agilent Technologies, Santa Clara, CA). For hematoxylin and eosin, periodic acid-Schiff, Prussian blue, and Sirius red staining, formalin-fixed, paraffin-embedded samples were processed according to standard protocols provided by the manufacturer. Sections were imaged with ECLIPSE Ci microscope (Nikon, Alzenau, Germany), Axio (Zeiss, Jena, Germany), or ECLIPSE Ni-E microscope (Nikon). Processing of images was performed by NIS-Elements Imaging Software AR 5.02.00 (Nikon) and ImageJ software version 1.52e (https:// imagej.nih.gov/ij; NIH, Bethesda, MD).

\section{Antibodies}

Primary antibodies were as follows: mouse anti-BrdU monoclonal antibody (5292; Cell Signaling Technology, Frankfurt am Main, Germany), mouse anti-Ki-67 antibody (550609; BD Biosciences), rat anti-Ki-67 (14-5698-82; eBioscience, San Diego, CA), goat anti-CD32 (AF1460; R\&D Systems, Minneapolis, MN), goat anti-lymphatic vessel endothelial hyaluronan receptor (Lyve1) (AF2125; R\&D Systems), rat antiendomucin (14-5851-82; Thermo Fisher Scientific, Waltham, MA), rabbit anti-intercellular adhesion molecule 1 (10020-1AP; Proteintech, Rosemont, IL), rabbit antidesmin (ab15200; Abcam, Cambridge, UK), rabbit anti-glutamine synthetase
(G2781; Sigma-Aldrich, Taufkirchen, Germany), goat anti-arginase I (sc-18351; Santa Cruz Biotechnology, Dallas, TX), goat anti-Rhbg (PA5-19369; Thermo Fisher Scientific), rabbit anti-phosphorylated c-Met (Tyr1234/1235; 3077; Cell Signaling Technology), mouse anti-c-Met (3127; Cell Signaling Technology), mouse anti-Hgf (NBP1-19182; Novus Biologicals, Centennial, CO), mouse anti-glyceraldehyde-3phosphate dehydrogenase (Gapdh; sc-32233; Santa Cruz Biotechnology), mouse anti- $\beta$-actin (sc-69879; Santa Cruz Biotechnology), and mouse anti-Deptor (A-3; SC-398169; Santa Cruz Biotechnology). Secondary antibodies were as follows: anti-mouse IgG horse radish peroxidase (HRP) conjugate (W402B; Promega, Fitchburg, WI), anti-rabbit IgG HRP conjugate (W401B; Promega), and EnVision-System-HRP-labeled polymer anti-mouse (K4001; Dako, Agilent Technologies); Alexa-Fluor 488, Alexa-Fluor 647, and Cy3-conjugated secondary antibodies were purchased from Dianova (Hamburg, Germany).

\section{RNA in Situ Hybridization}

Liver tissue was divided into sections (4 $\mu \mathrm{m}$ thick). A modified nonisotopic in situ hybridization protocol was performed using the BaseScope Reagent Kit v2-RED and BaseScope Duplex Reagent Kit (323900 and 323800; Advanced Cell Diagnostics, Newark, CA) following the manufacturer's recommended protocol. Specific probes against the positive control mouse Ppib (BaseScope Positive Control; Mm-PPIB-3ZZ; Advanced Cell Diagnostics), mouse Hgf (BA-Mm-Hgf-2zz-st; Nm_001289458.1; 692 823; Advanced Cell Diagnostics), and mouse Stab2 (BAMm-Stab2-2EJ-C2; 4zz targeting 137-510 of NM_138673.3) were applied. Finally, sections were stained with BaseScope Fast RED and BaseScope Green and counterstained with hematoxylin. Quantification of in situ hybridization staining was performed by QuPath 0.1.2 software (University of Edinburgh, Scotland, UK). ${ }^{31}$ RNAscope Multiplex Fluorescent in situ hybridization (FISH) version 2 assay (323110; Advanced Cell Diagnostics) was performed with mouse Deptor-Channel 3 (261-1458 NM_145470.3; Advanced Cell Diagnostics), mouse Hgf-Channel 3 (1120-2030 NM_010427.4;

Table 2 Deptor siRNA Sequence Information

\begin{tabular}{lll}
\hline Deptor siRNA no. & Sense & Antisense \\
\hline 1 & $5^{\prime}$-GCACCUUCCCAUUGGAUAATT-3' & 5'-UUAUCCAAUGGGAAGGUGCCG-3' $^{\prime}$ \\
2 & $5^{\prime}$-CAAACUGUUUUGUCGCAAATT-3' & $5^{\prime}$-UUUGCGACAAAACAGUUUGGG-3' \\
3 & $5^{\prime}$-CCCAUUUGUGGACAGCAAUTT-3' & $5^{\prime}$-AUUGCUGUCCACAAAUGGGTG-3' \\
\hline
\end{tabular}


A

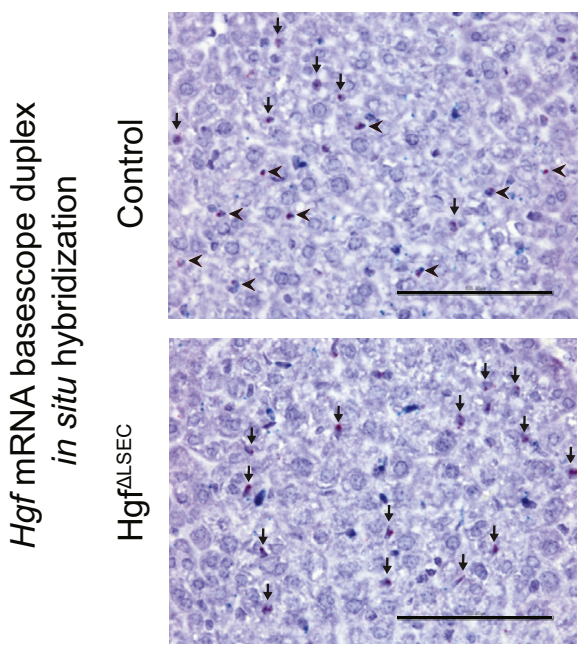

B

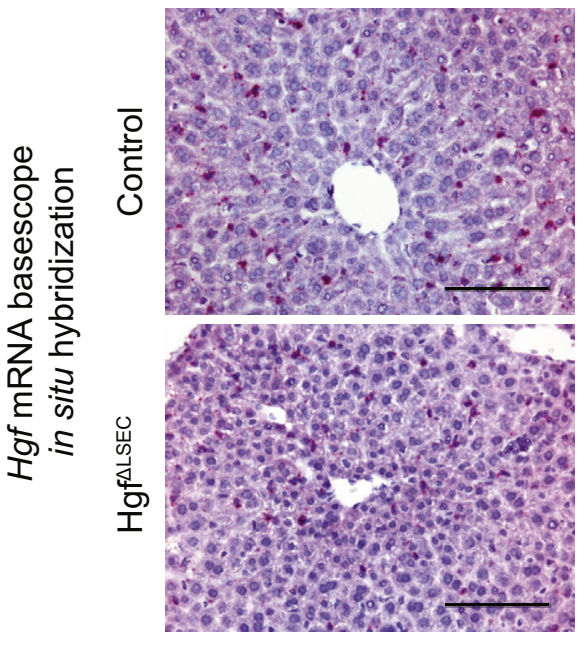

C
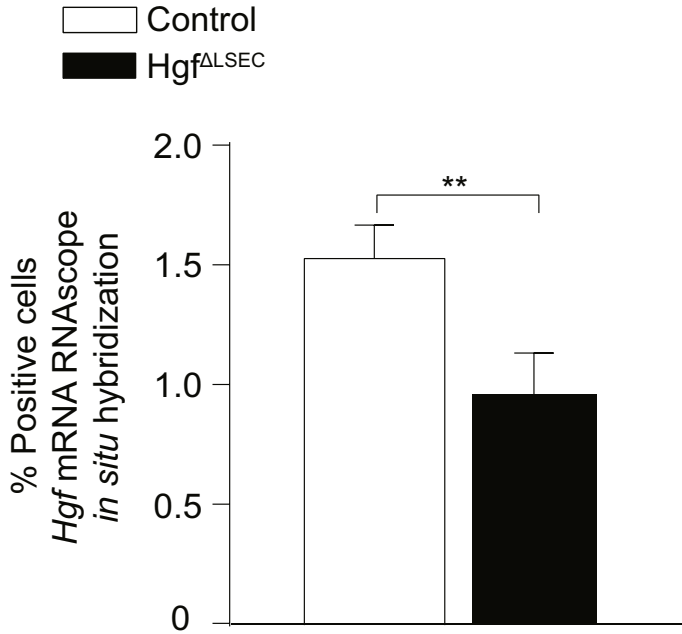

Figure $1 H g f$ mRNA is reduced in livers of $\mathrm{Hgf}^{\Delta \mathrm{LSEC}}$ mice. A: $\mathrm{Hgf}$ (green) and Stab2 (red) mRNA BaseScope Duplex in situ hybridization assay. Arrowheads indicate $\mathrm{Hgf} / \mathrm{Stab2}$ double-positive liver sinusoidal endothelial cells (LSECs); arrows, Hgf-negative, Stab2-positive LSECs (female). B: $\mathrm{Hgf}$ mRNA BaseScope in situ hybridization (female). C: Quantification of in situ hybridization of liver tissues of control and $\mathrm{Hgf}^{\triangle \mathrm{LSEC}}$ mice. $n=5(\mathbf{A})$; $n=3(\mathbf{B}) .{ }^{*} P<0.01$. Scale bars $=100 \mu \mathrm{m}(\mathbf{A}$ and $\mathbf{B})$.
Advanced Cell Diagnostics), and mouse Stab2, CD68, and Desmin (4249 - $5075 \quad$ NM_138673.2, 2-1007 NM_009853.1, and 896-1859 NM_010043.1; Advanced Cell Diagnostics) probes, as recommended by the manufacturer. Images were acquired by fluorescence microscopy.

\section{Quantitative RT-PCR}

RNA from snap-frozen liver tissues was extracted using RNeasy Plus Mini Kit (Qiagen, Hilden, Germany). cDNA was synthesized with QuantiTect Reverse Transcription Kit (Qiagen). Primers were designed using Primer-BLAST ( $h t t p: / / w w w$. ncbi.nlm.nih.gov/tools/primer-blast; last accessed August 20, 2019) and PrimerBank (https://pga.mgh.harvard.edu/ primerbank; last accessed August 22, 2019). All the primers used in the present study are listed in Table 1. Quantitative RT-PCR was performed in a LightCycler 480 real-time PCR machine (Roche, Mannheim, Germany) using KAPA SYBR FAST Kit (KK4611; Sigma-Aldrich). Relative mRNA expression was quantified by normalizing against Gapdh.

\section{Western Blot Analysis}

All liver tissues were lysed from snap-frozen liver using $1 \times$ radioimmunoprecipitation assay buffer (Cell Signaling Technology), and homogenates were spun at full speed for 20 minutes at $4^{\circ} \mathrm{C}$ to remove cell debris. Aliquots of protein lysate $(20 \mu \mathrm{g})$ were subjected to SDS-PAGE, followed by transfer onto nitrocellulose membrane and incubation with primary antibodies. Then, the membranes were incubated with a horseradish peroxidase-conjugated secondary antibody and developed using ECL Western Blotting Detection reagents (GE Healthcare, Amersham, UK) and SuperSignal West Femto Substrate (Thermo Fisher Scientific). Relative protein expression levels were analyzed using the ImageJ software version $1.52 \mathrm{e}$.

\section{Blood Parameters and Enzyme-Linked Immunosorbent Assay}

Plasma was collected from female $\mathrm{Hgf}^{\Delta \mathrm{LSEC}}$ and control mice at the age of 2 months and analyzed for the following routine parameters: alanine aminotransferase (ALT), aspartate aminotransferase (AST), glutamate dehydrogenase $(\mathrm{GLDH}), \mathrm{Na}, \mathrm{K}, \mathrm{Ca}, \mathrm{PO}_{4}$, glucose, protein, and urea (cobas c 311 analyser; Roche). Serum ALT in PH mice was detected by enzyme linked immunosorbent assay kit for alanine aminotransferase (Cloud-Clone Corp., Rockville, $\mathrm{MD}$ ), according to manufacturer instructions.

\section{RNA Sequencing}

Rawcount matrices were imported into $\mathrm{R}$ (The R Foundation for Statistical Computing, Vienna, Austria), and differential gene expression analysis was conducted with DESeq2. ${ }^{32}$ Dispersion estimates were calculated setting the 
Table 3 Mendelian Frequency of Stab2-iCre ${ }^{t g / w t} ; \mathrm{Hg} f^{f l / f l}$ Mice

\begin{tabular}{|c|c|c|c|}
\hline Genotype & Mice, $n$ & $\begin{array}{l}\text { Mendelian } \\
\text { frequency, \% }\end{array}$ & Observed, \% \\
\hline$\overline{H g f t / w t}$ & 8 & 25 & 22.2 \\
\hline$H g f^{f / / f l}$ & 10 & 25 & 27.8 \\
\hline Stab2-iCres ${ }^{t g / w t} ; H g f^{f l / w t}$ & 10 & 25 & 27.8 \\
\hline Stab2-iCre ${ }^{t g / w t} ; H g f^{f / f l}$ & 8 & 25 & 22.2 \\
\hline
\end{tabular}

option fitType to parametric using all samples available. A Wald test was conducted for detecting differences between genotypes for all available time points. A gene was determined to be significantly regulated if $P<0.05$. Genes being regulated at time point 48 hours are shown as heat map, together with samples collected at the 0 -hour time point.

\section{Statistical Analysis}

All statistics were performed using the GraphPad Prism 7.0 (GraphPad, San Diego, CA) and SigmaPlot 11 Software (Systat Software GmbH, Erkrath, Germany). All data are presented as means \pm SEM. Statistical differences were analyzed using the two-tailed unpaired $t$-test or the $U$-test and $\chi^{2}$ test. Statistical significance was set at $P<0.05$.

\section{Cell Lines and Cell Culture}

All cell culture experiments were performed using the human hepatocellular carcinoma cell line Huh7 and the mouse hepatocyte cell line AML-12, purchased from ATCC (Manassas, VA). Huh7 cells were cultured in Dulbecco's modified Eagle's medium (Thermo Fisher Scientific), $1 \%$ glucose supplemented with $10 \%$ fetal bovine serum, and $1 \%$ penicillin/streptomycin. AML-12 cells were cultured in Dulbecco's modified Eagle's medium/F-12 medium (Thermo Fisher Scientific), 1\% glucose supplemented with $10 \%$ fetal bovine serum, and $1 \%$ penicillin/ streptomycin. All cells were cultivated in $5 \% \mathrm{CO}_{2}$ at $37^{\circ} \mathrm{C}$.

\section{siRNA Transfection}

Huh7 cells were seeded freshly. After a cell confluence of $30 \%$ to $50 \%$, siRNA transfection was performed using Lipofectamine RNAiMAX Reagent (Invitrogen, Waltham, MA), according to the manufacturer's instructions. Three different siRNAs (Table 2) constructs targeting Deptor
A
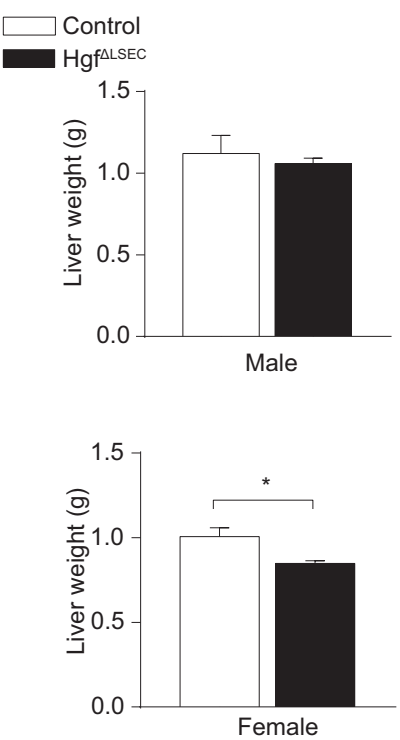

B
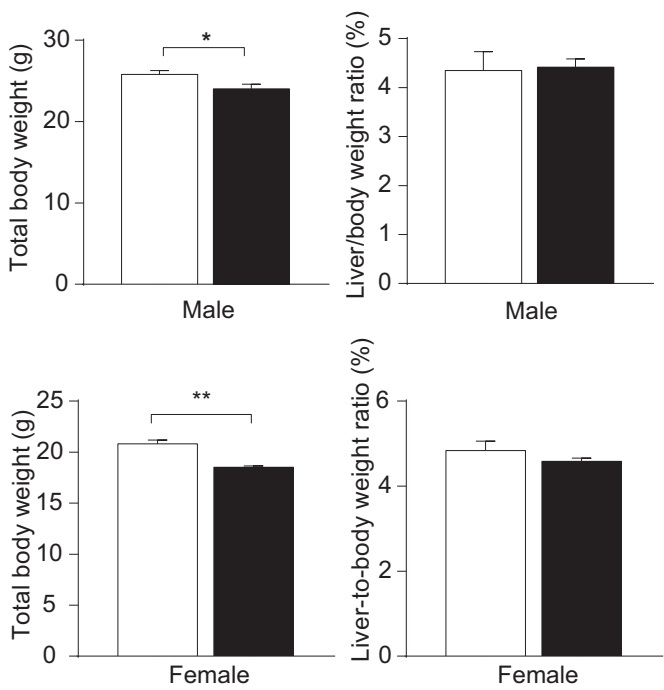

Figure $2 \quad H g f$ ablation in liver sinusoidal endothelial cells (LSECS) results in reduced organismal growth. A: Liver weight, total body weight, and liver/body weight ratio of 9-week -old control and $\mathrm{Hgf}^{\Delta{ }^{\mathrm{LECC}}}$ mice (male and female). B: Spleen weight and spleen/body weight ratio of 9-week-old control and $\mathrm{Hgf}^{\mathrm{ASEC}}$ mice (male and female). Data are expressed as means \pm SEM (A and $\mathbf{B}) . n \geq 5$ (A and $\mathbf{B}) .{ }^{*} P<0.05,{ }^{* *} P<0.01$. 
A
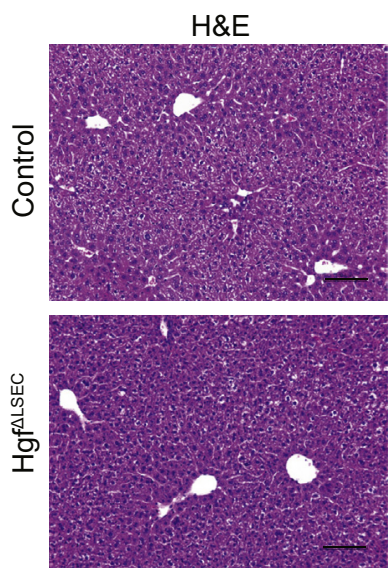

B

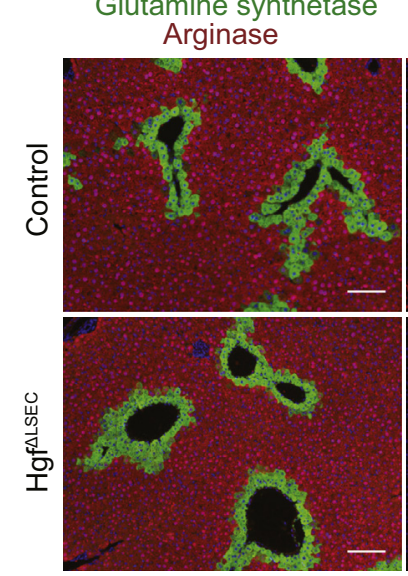

Arginase

C

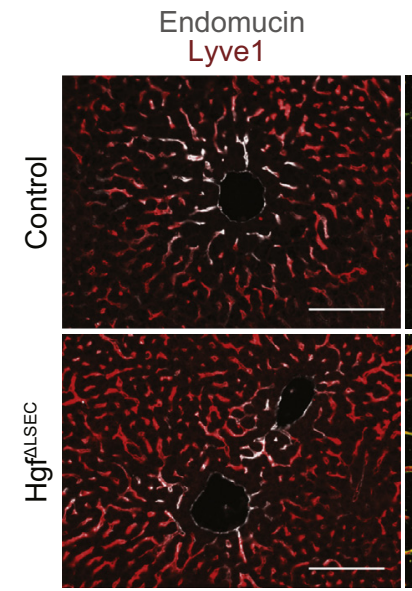

PAS
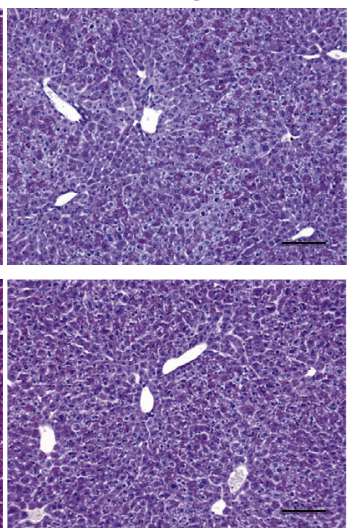

Rhbg
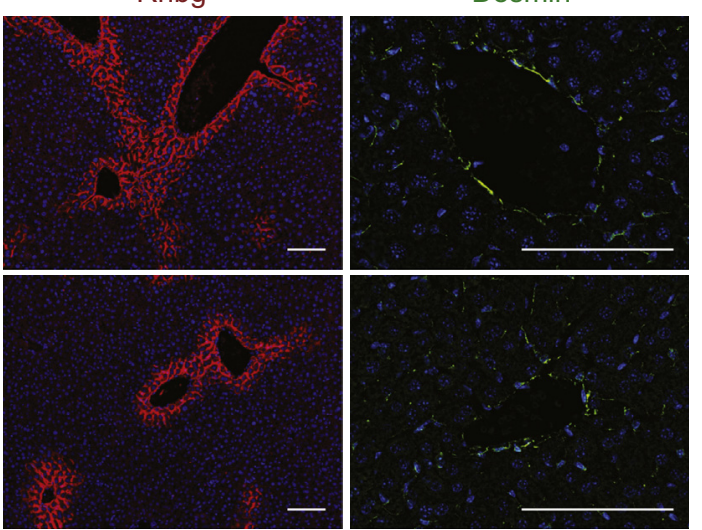

Icam1
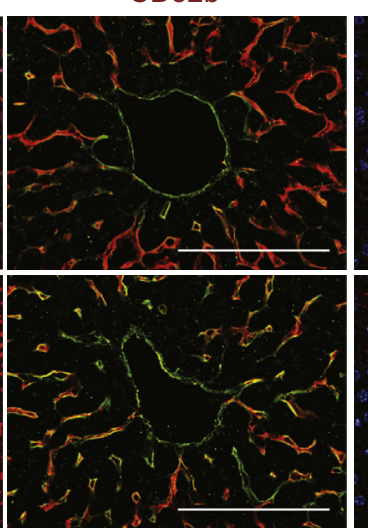

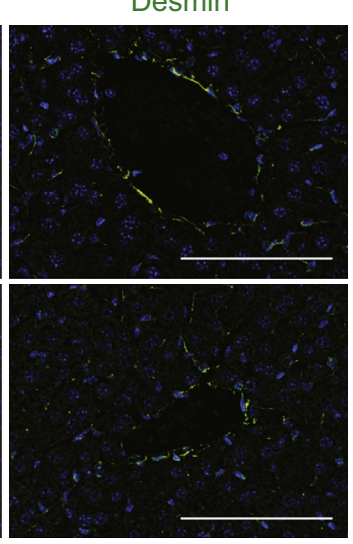

Sirius red
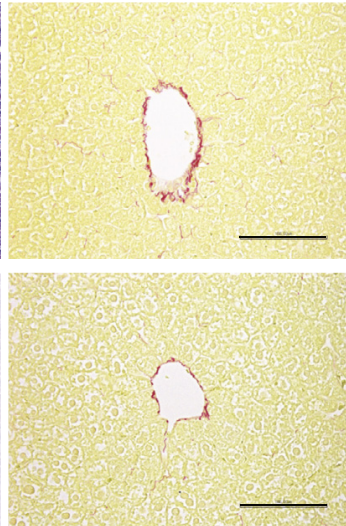

(20smin

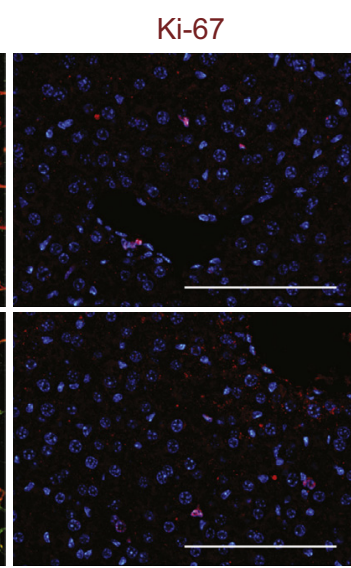

Figure $3 \quad H g f$ ablation in liver sinusoidal endothelial cells (LSECS) does not impair liver morphology. A: Hematoxylin and eosin (H\&E), periodic acid-Schiff (PAS), and Sirius red staining of liver of $\mathrm{Hgf}^{\triangle \mathrm{LSEC}}$ mice compared with controls (female). B: Immunofluorescence of glutamine synthetase (GS) and arginase, Rhbg, and Desmin. C: Lymphatic vessel endothelial hyaluronan receptor 1 (Lyve1) and endomucin, intercellular adhesion molecule 1 (Icam1) and CD32, and Ki-67 in liver of female control and $\mathrm{Hgf}^{\triangle \mathrm{LSEC}}$ mice. $n=5$ (A, B, left and middle columns, and C, left column); $n=3$ (B, right column, and $C$, middle and right columns). Scale bars $=100 \mu \mathrm{m}(\mathbf{A}-\mathbf{C})$.
(Thermo Fisher Scientific) were used. All experiments were performed with a negative control (Thermo Fisher Scientific). Huh7 cells were harvested 48 hours after transfection for RNA and protein analyses.

\section{Stimulated Assay}

Control and siRNA-transfected Huh7 cells were grown and serum starved for 12 hours, followed by treatment with murine Hgf (mHGF) (R\&D Systems).

\section{Growth Curves}

Cell proliferation was determined by performing growth curves using the Neubauer-improved counting chamber (Sigma-Aldrich) to count siRNA transfected and untransfected cells. After siRNA transfection, an equal number of cells was seeded for each group of cells. As soon as the first group of cells of Huh7 cells reached a cell confluence of $80 \%$ to $90 \%$, all groups of the cell line were counted and seeded again in an equal number of cells. 


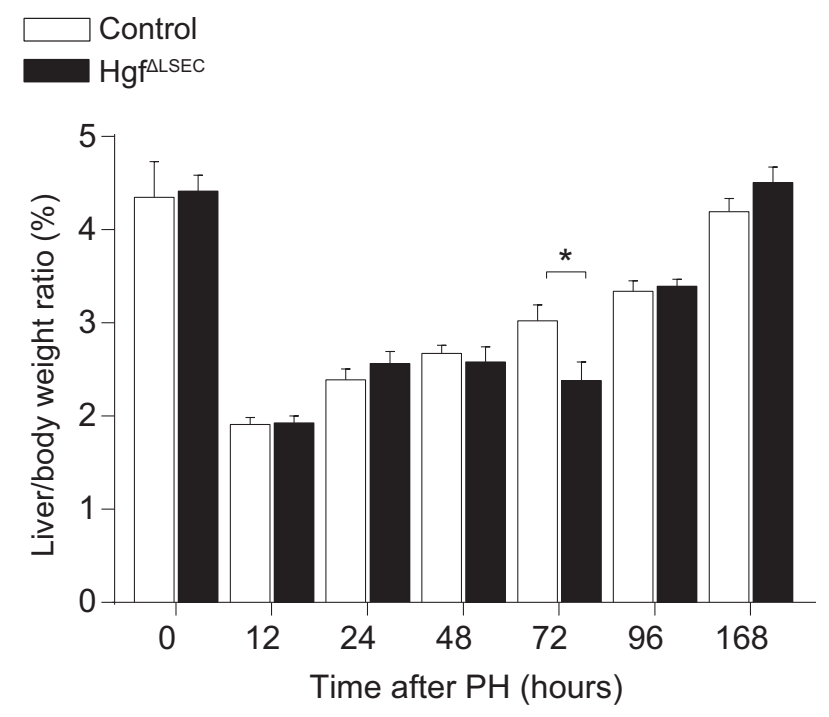

Figure 4 Kinetics of liver/body weight ratios after partial hepatectomy (PH). Liver/body weight ratio of $\mathrm{Hgf}^{\Delta \mathrm{LSEC}}$ and control mice at different time points after $70 \% \mathrm{PH}$. Data are expressed as means \pm SEM. ${ }^{*} P<0.05$.

\section{Results}

Hgf Ablation in LSECs Results in Reduced Organismal Growth but Normal Liver Development

In the liver, $H g f$ expression is described in LSECs, Kupffer cells, and stellate cells, and expression of $H g f$ in these cell types was confirmed by colocalization of $\mathrm{Hg} f$ with $\mathrm{Stab2}^{+}$ cells (LSECs), CD68 ${ }^{+}$cells (Kupffer cells), and Desmin ${ }^{+}$ cells (stellate cells) by co-fluorescent in situ hybridization (Supplemental Figure S1A). To analyze the role of LSECderived Hgf in liver physiology and regeneration, homozygous Hgf ${ }^{\text {ex.5 flox }}\left(H g f^{f / f l}\right)$ mice $^{29}$ were crossed with mice with Stab2 promoter-driven Cre expression (Stab2-iCre) ${ }^{16}$ to generate $\mathrm{Stab2}-\mathrm{iCr} e^{t g / w t} ; \mathrm{Hg} f^{f / f t}\left(\mathrm{Hgf}^{\Delta \mathrm{LSEC}}\right)$ with specific deletion of $\mathrm{Hgf}$ in LSECs but no other hepatic cells (Figure 1A). $\mathrm{Hgf}^{\Delta \mathrm{LSEC}}$ embryos did not show any gross developmental defects at $\mathrm{E} 14.5,^{28}$ were born in normal mendelian frequency, and survived to late adulthood (Table 3). $H g f$ expression was significantly reduced in the adult liver of $\mathrm{Hgf}^{\triangle \mathrm{LSEC}}$ (Figure 1, B and $\mathrm{C}$, and Supplemental Figure S1B).

Despite no apparent macroscopic differences, $\mathrm{Hgf}^{\Delta \mathrm{LSEC}}$ mice had lower body weights in comparison to control $H g f^{f /}$ ${ }^{f t}$ or $H g f^{f / w t}$ mice (Figure 2A and Supplemental Figure S2A). Despite lower liver weights in $\mathrm{Hgf}^{\Delta \mathrm{LSEC}}$ mice, liver/body ratio was unaltered in both sexes (Figure 2A). Similarly, spleen/body weight ratios did not differ, whereas only male $\mathrm{Hgf}^{\Delta \mathrm{LSEC}}$ mice had lower total spleen weights in comparison to control mice (Figure 2B).

Routine histology (hematoxylin and eosin, periodic acid-Schiff, and Sirius red) of $\mathrm{Hgf}^{\mathrm{\Delta LSEC}}$ mice did not reveal significant morphologic changes, inflammation, or depositions of polysaccharides and collagens (Figure 3A).
Metabolic liver zonation was maintained, as demonstrated by regular expression of glutamine synthetase, arginase, and $\mathrm{Rhbg}$ in $\mathrm{Hgf}^{\triangle \mathrm{LSEC}}$ (Figure 3B). Expression of endomucin (Emcn)/intercellular adhesion molecule 1 in pericentral LSECs and Lyve-1/CD32 in midzonal LSECs was present in $\mathrm{Hgf}^{\triangle \mathrm{LSEC}}$ mice, indicating proper sinusoidal endothelial differentiation and zonation (Figure 3C). Likewise, Desmin $^{+}$stellate cells were not altered in size or number, and the proliferation of hepatic cells, as assessed by $\mathrm{Ki}-67$ staining, was not altered (Figure 3, B and C). In addition, liver enzymes (AST and ALT), electrolytes, and basic metabolic parameters, as assessed by serum analysis, revealed comparable levels in $\mathrm{Hgf}^{\triangle \mathrm{LSEC}}$ and control mice (Supplemental Figure S2B). Therefore, except for being slightly but significantly lighter, $\mathrm{Hgf}^{\Delta \mathrm{LSEC}}$ did not show major alterations of liver development and function or general impairment.

\section{Liver Regeneration Is Compromised in $\mathrm{Hgf}^{\Delta \mathrm{LSEC}}$ Mice}

To further elucidate the role of angiocrine Hgf signaling in liver regeneration, $70 \% \mathrm{PH}$ was performed in both $\mathrm{Hgf}^{\Delta \mathrm{LSEC}}$ and control mice. $\mathrm{Hgf}^{\Delta \mathrm{LSEC}}$ mice showed a significantly reduced liver/body weight ratio compared with the control group at 72 hours after hepatectomy (Figure 4). Typically, proliferation of all hepatic cells sharply increases after $\mathrm{PH}$, peaking at 48 to 72 hours after operation. ${ }^{33}$ Therefore, hepatocytes that enter into the Sphase of the cell cycle were examined by incorporation of BrdU. Staining for BrdU revealed that hepatocyte proliferation in control mice started at 24 hours, reaching a peak at 48 hours, and terminating at 168 hours after $\mathrm{PH}$ (Figure 5A). In contrast, the fraction of BrdU-positive hepatocytes in $\mathrm{Hgf}^{\triangle \mathrm{LSEC}}$ mice was significantly lower at 48 hours after $\mathrm{PH}$ (Figure 5B). Consistent with this finding, $\mathrm{Hgf}^{\triangle \mathrm{LSEC}}$ mouse livers displayed fewer $\mathrm{Ki}$ 67-positive hepatocytes at this time point (Figure 5B), whereas assessment of hepatocyte sizes in control and $\mathrm{Hgf}^{\triangle \mathrm{LSEC}}$ livers at 48,72 , and 96 hours after PH did not show any significant changes (Supplemental Figure S3A). These findings indicate that the regenerative capacity of the liver may be compromised in $\mathrm{Hgf}^{\Delta \mathrm{LSEC}}$ mice.

After $\mathrm{PH}$, mice were carefully and regularly observed for signs of pain or distress. As surrogate marker for lethality, it was analyzed how many mice reached the predefined criteria of distress (Materials and Methods) and had to be euthanized. This only occurred in $\mathrm{Hgf}^{\mathrm{DLSEC}}$ but not control mice after PH (Supplemental Table S1). All control mice were healthy and survived up to the designated time points after $\mathrm{PH}$, but $12.8 \%$ of $\mathrm{Hgf}^{\Delta \mathrm{LSEC}}$ mice reached the predefined criteria of distress 48 to 72 hours after PH (Supplemental Table S1). Assessment of liver histology revealed that a subset of $\mathrm{Hgf}^{\triangle \mathrm{LSEC}}$ mice had liver necrosis 48 to 96 hours after $\mathrm{PH}$, but no necrotic area was found in control mice after PH (Figure 6, A and B, and Supplemental Table S2). Necrosis showed a random 

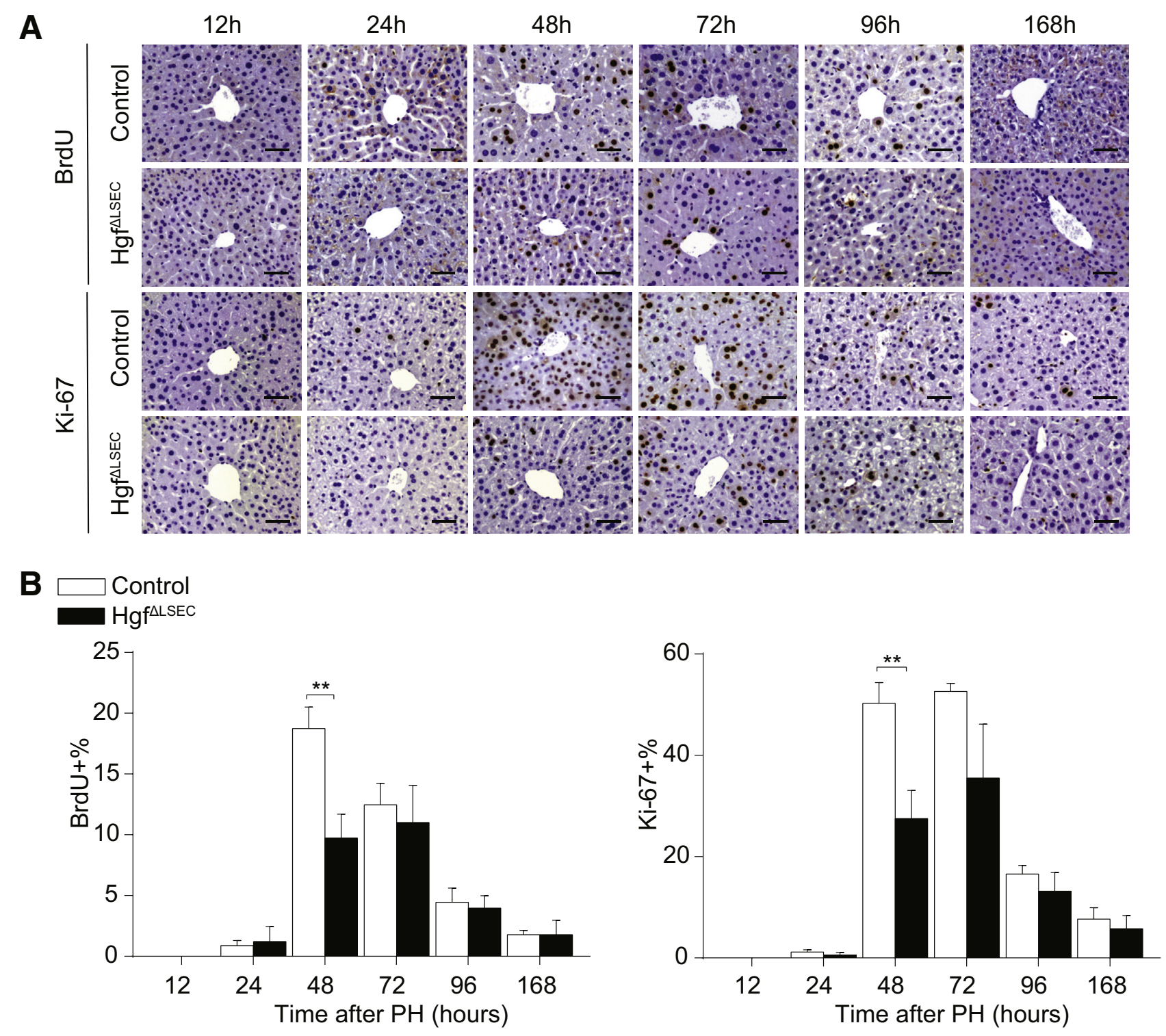

Figure 5 Hepatocyte proliferation is compromised in $\mathrm{Hgf}^{\mathrm{ALSEC}}$ mice in response to partial hepatectomy (PH). A: Representative micrographs of liver sections immunostained with bromodeoxyuridine (BrdU) and Ki-67 antibody from control and $\mathrm{Hgf}^{\Delta \mathrm{LSEC}}$ mice after $70 \% \mathrm{PH}$. B: Quantification of BrdU- and Ki67-positive hepatocytes at different time points after $70 \%$ PH. Data are expressed as means \pm SEM (B). $n \geq 5\left(\right.$ A). ${ }^{* * P}<0.01$. Scale bars $=200 \mu \mathrm{m}(\mathbf{A})$.

distribution throughout the liver and covered an area of $15 \%$ to $35 \%$ per liver section (Supplemental Table S3). It was accompanied by a scarce infiltration of neutrophilic granulocytes in some necrotic areas (Supplemental Figure S3B). Serum ALT was measured at different time points after $\mathrm{PH}$, and significantly higher ALT levels were found at 48 and 168 hours after PH in $\mathrm{Hgf}^{\Delta \mathrm{LSEC}}$ compared with control mice (Figure 6C). Furthermore, comparison of ALT in necrotic $\mathrm{Hgf}^{\mathrm{ALSEC}}$ livers with nonnecrotic $\mathrm{Hgf}^{\triangle \mathrm{LSEC}}$ and control livers showed significantly higher ALT levels in necrotic $\mathrm{Hgf}^{\triangle \mathrm{LSEC}}$ livers (Figure 6D). Overall, the findings of increased lethality, reduced hepatocyte proliferation, and enhanced liver necrosis demonstrate that the regenerative capacity of $\mathrm{Hgf}^{\triangle \mathrm{LSEC}}$ is impaired after $70 \% \mathrm{PH}$.
Angiocrine Activation of the $\mathrm{Hgf} / \mathrm{c}-$ Met Axis after $\mathrm{PH}$ Is Impaired in $\mathrm{Hgf}^{\Delta L S E C}$ and Results in Decreased Induction of Deptor and Other Potential Regulators of Hepatocyte Proliferation

The kinetics of Hgf expression and c-Met activation during liver regeneration were analyzed. $H g f$ mRNA increased rapidly in control but not $\mathrm{Hgf}^{\mathrm{LLSEC}}$ mice 12 hours after $\mathrm{PH}$, as observed by quantitative RT-PCR analysis of whole liver lysates (Figure 7A). Although there was a trend toward lower $H g f$ mRNA expression in $\mathrm{Hgf}^{\triangle \mathrm{LSEC}}$ mice, no further significant differences were observed between the two groups at other time points (Figure 7A and Supplemental Figure $\mathrm{S} 3 \mathrm{C}$ ). On the protein level, there was a similar trend at all time points, with significantly lower $\mathrm{Hgf}$ protein 
A
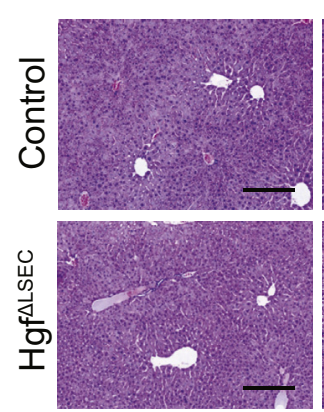

B
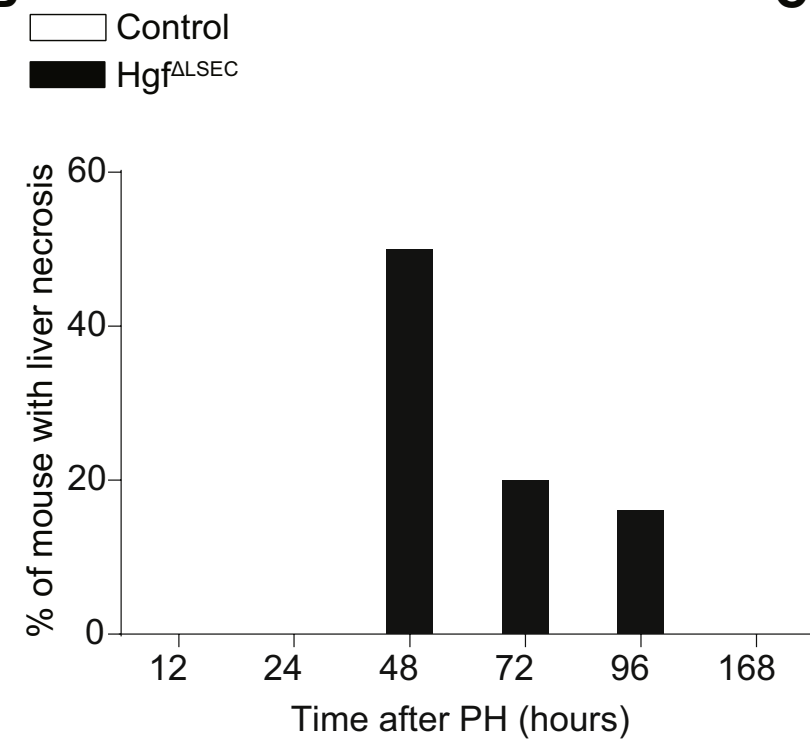

D
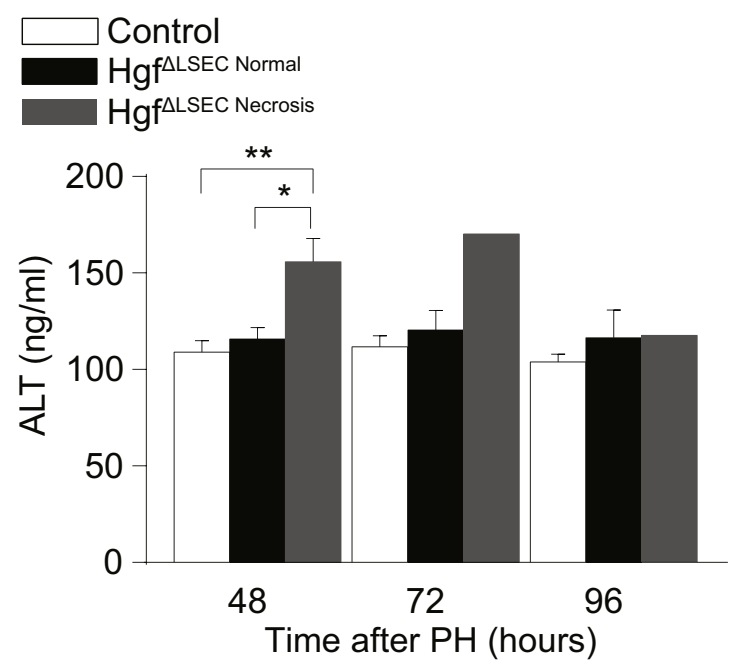

levels 48 and 72 hours after $\mathrm{PH}$ in $\mathrm{Hgf}^{\Delta \mathrm{LSEC}}$ mice (Figure 7, $\mathrm{B}$ and $\mathrm{C}$ ). Although $\mathrm{Hgf}^{\Delta \mathrm{LSEC}}$ had lower levels of $\mathrm{Hgf}$, control and $\mathrm{Hgf}^{\Delta \text { LSEC }}$ both had significantly higher levels of Hgf protein 12 to 72 hours after PH (Figure 7, B and C). Phosphorylation of c-Met was similarly regulated as $\mathrm{Hgf}$

C
$72 \mathrm{~h}$
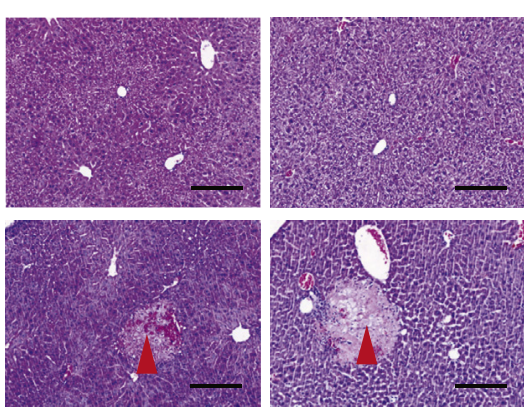

$168 \mathrm{~h}$

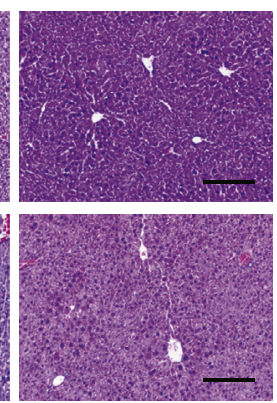

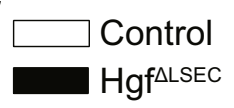

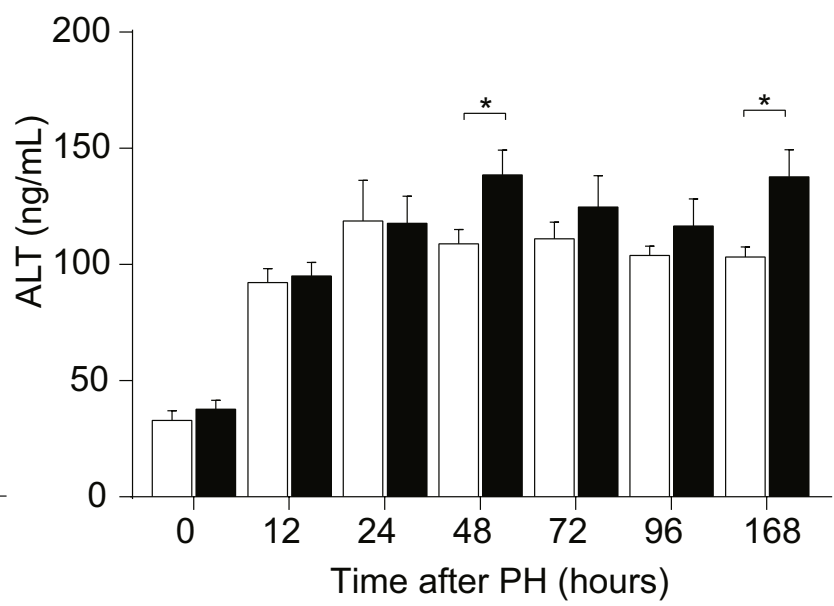

Figure $6 \quad H g f$ ablation in liver sinusoidal endothelial cells (LSECS) leads to tissue necrosis on partial hepatectomy (PH). A: Hematoxylin and eosin staining of liver sections of control and $\mathrm{Hgf}^{\Delta \mathrm{LSEC}}$ mice at different time points after $70 \% \mathrm{PH}$. Arrowheads show necrotic areas of $\mathrm{Hgf}^{\triangle \mathrm{LSEC}}$ mice livers after $70 \% \mathrm{PH}$. B: Percentage of necrotic areas of liver sections from $\mathrm{Hgf}^{\Delta \mathrm{LEC}}$ mice after PH. C: Alanine aminotransferase (ALT) measurement in sera from control and $\mathrm{Hgf}^{\triangle L S E C}$ mice after PH. D: ALT levels separately measured in sera from control, $\mathrm{Hgf}^{\triangle \mathrm{LSEC}}$ mice with liver necrosis and without liver necrosis, at 48, 72, and 96 hours after PH. Data are expressed as means \pm SEM (C and D). $n \geq 5$ (A and C). ${ }^{*} P<0.05,{ }^{*} P<0.01$. Scale bars $=100 \mu \mathrm{m}(\mathbf{A})$.

protein and was significantly lower in $\mathrm{Hgf}^{\mathrm{ALSEC}}$ at 48 hours after PH. Total c-Met did not differ during the different phases of regeneration nor did it differ between control and $\mathrm{Hgf}^{\triangle \mathrm{LSEC}}$ mice (Figure 7, B and C). Therefore, LSECderived $\mathrm{Hgf}$ controlled the activation dynamics of the Hgf/ 

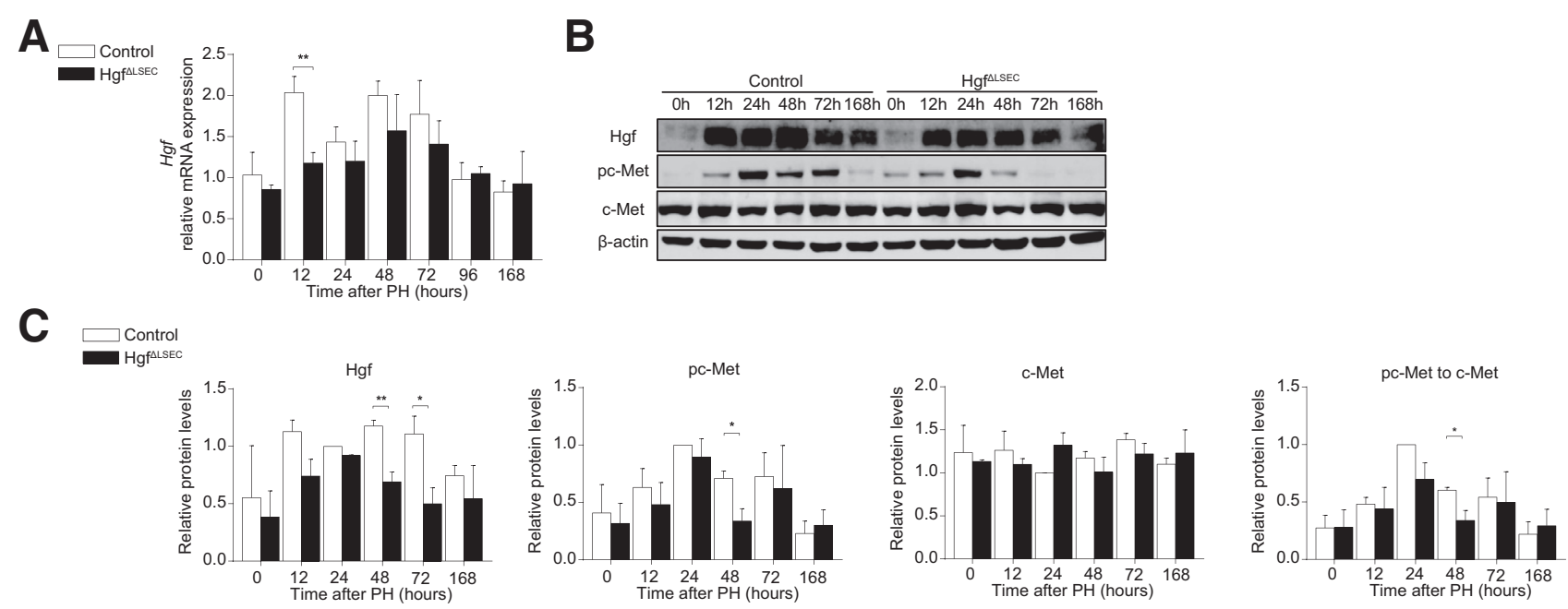

Figure $7 \mathrm{Hgf} / \mathrm{c}-$ Met signaling pathway is impaired in $\mathrm{Hgf}^{\Delta \mathrm{LSEC}}$ mice after partial hepatectomy (PH). A: Quantitative RT-PCR analysis of $\mathrm{Hgf}$ in livers of control and $\mathrm{Hgf}^{\Delta \mathrm{LSEC}}$ mice at different time points after $70 \% \mathrm{PH}$. Gapdh was used as housekeeping gene. B: Representative immunoblots of Hgf in livers of control and $\mathrm{Hgf}^{\triangle \mathrm{LSEC}}$ mice at different time points after $70 \% \mathrm{PH}$. C: Quantification of hepatic expression of Hgf/c-Met signaling pathway after $70 \% \mathrm{PH}$ in control and Hgf ${ }^{\Delta L E C C}$ mice. $n=5(\mathbf{A}) ; n=3$ (B). ${ }^{*} P<0.05,{ }^{* *} P<0.01$.

c-Met axis. Loss of angiocrine Hgf caused the strongest impairment of this activation 48 hours after $\mathrm{PH}$.

To decipher the molecular alterations that result from the impairment of angiocrine activation of the $\mathrm{Hgf} / \mathrm{c}-\mathrm{Met}$ axis in liver regeneration, RNA sequencing was performed on whole liver RNA from control and $\mathrm{Hgf}^{\Delta \mathrm{LSEC}}$ mice at 0 and 48 hours after PH (Figure 8A). Before PH (0 hour), $\mathrm{Hgf}^{\Delta \mathrm{LSEC}}$ and control livers did not exhibit any differentially expressed gene. However, 48 hours after $\mathrm{PH}$, the expression of 17 genes was differentially regulated in $\mathrm{Hgf}^{\triangle L S E C}$ and control livers. Although 12 genes were upregulated, seven of these genes were strongly up-regulated only in $\mathrm{Hgf}^{\triangle \mathrm{LSEC}}$ livers that contained significant necrosis (Figure 8A). The remaining five genes (ie, Rps24, Gm9843, $S d s l, G m 16157$, and Rps2) were up-regulated in $\mathrm{Hgf}^{\mathrm{DLSEC}}$ independently of the presence of necrosis. Five genes (ie, Bboxl, Cyp27al, Deptor, Agmat, and Ccdc152) were significantly down-regulated in $\mathrm{Hgf}^{\triangle \mathrm{LSEC}}$. Differential expression of Deptor was confirmed on the protein level by Western blot analysis and fluorescence in situ hybridization (Figure 8, B and C). Herein, $\mathrm{Hgf}^{\triangle \mathrm{LSEC}}$ showed a fourfold lower expression of hepatic Deptor at 48 hours after PH in comparison to control (Figure 8B). Furthermore, Deptor is predominantly expressed in hepatocytes and showed the strongest reduction in the perinecrotic areas of $\mathrm{Hgf}^{\mathrm{DLSEC}}$ livers (Figure 8C). Hgf stimulation of AML cells did not appear to directly induce Deptor mRNA expression, indicating that it is not directly regulated by $\mathrm{Hgf}$ in vitro (Supplemental Figure S4A). However, this may not exclude direct or indirect modulation of Deptor via Hgf during $\mathrm{PH}$ in vivo. Functionally, siRNA-mediated knockdown of Deptor decreased proliferation of Huh7 cells in vitro by approximately $50 \%$, supporting that Deptor is functionally involved in the reduced hepatocyte proliferation after $\mathrm{PH}$ in $\mathrm{Hgf}^{\Delta \mathrm{LSEC}}$ (Supplemental Figure S4, B and C).

\section{Discussion}

Proliferative events after $\mathrm{PH}$ are initiated and controlled by complete mitogens and auxiliary mitogens. Hgf and its receptor c-Met as well as epidermal growth factor receptor and its ligands represent so-called complete mitogens. ${ }^{34} \mathrm{Hgf}$ is known as a major hepatocyte mitogen and performs its activity through activation of the receptor tyrosine kinase cMet. Hgf has been shown to be essential for liver growth and organ regeneration. Although systematic ablation of Hgf or its receptor c-Met in mice leads to abnormal liver development and lethality in utero, ${ }^{8,9,35}$ hepatocyte-specific $c$-Met knockout mice are viable but have high mortality rates after $\mathrm{PH}^{26,36}$ Several studies were able to demonstrate the importance of the Hgf/c-Met signaling pathway during liver regeneration. ${ }^{29,37-39}$

As Hgf can be secreted by a wide range of cell types, there is increasing interest in the functional contributions of different cellular sources of Hgf. Recently, Cao and colleagues $^{27}$ used an inducible EC-specific $\mathrm{Hg}$ knockout mouse model to show that inducible deletion of endothelial $\mathrm{Hgf}$ impairs liver regeneration and enhances the susceptibility to profibrotic stimuli. Although inducible endothelial cell-specific knockout models are suitable for the analysis of transient effects of angiokine deprivation, constitutive cell-type-specific knockout models are needed to fully decipher its impact on development and long-term homeostasis. For example, induced conditional deletion of the Wnt cargo receptor Evi (Wls) in vascular endothelial-cadherin-positive endothelial cells of adult mice, described as being mosaic like, results in decreased expression of glutamine synthetase and Axin2 in pericentral hepatocytes without gross alterations of liver architecture. ${ }^{40}$ By using Stab2-Cre mice to delete Wls in LSECs, it has been shown that constitutive loss of angiocrine Wnt 


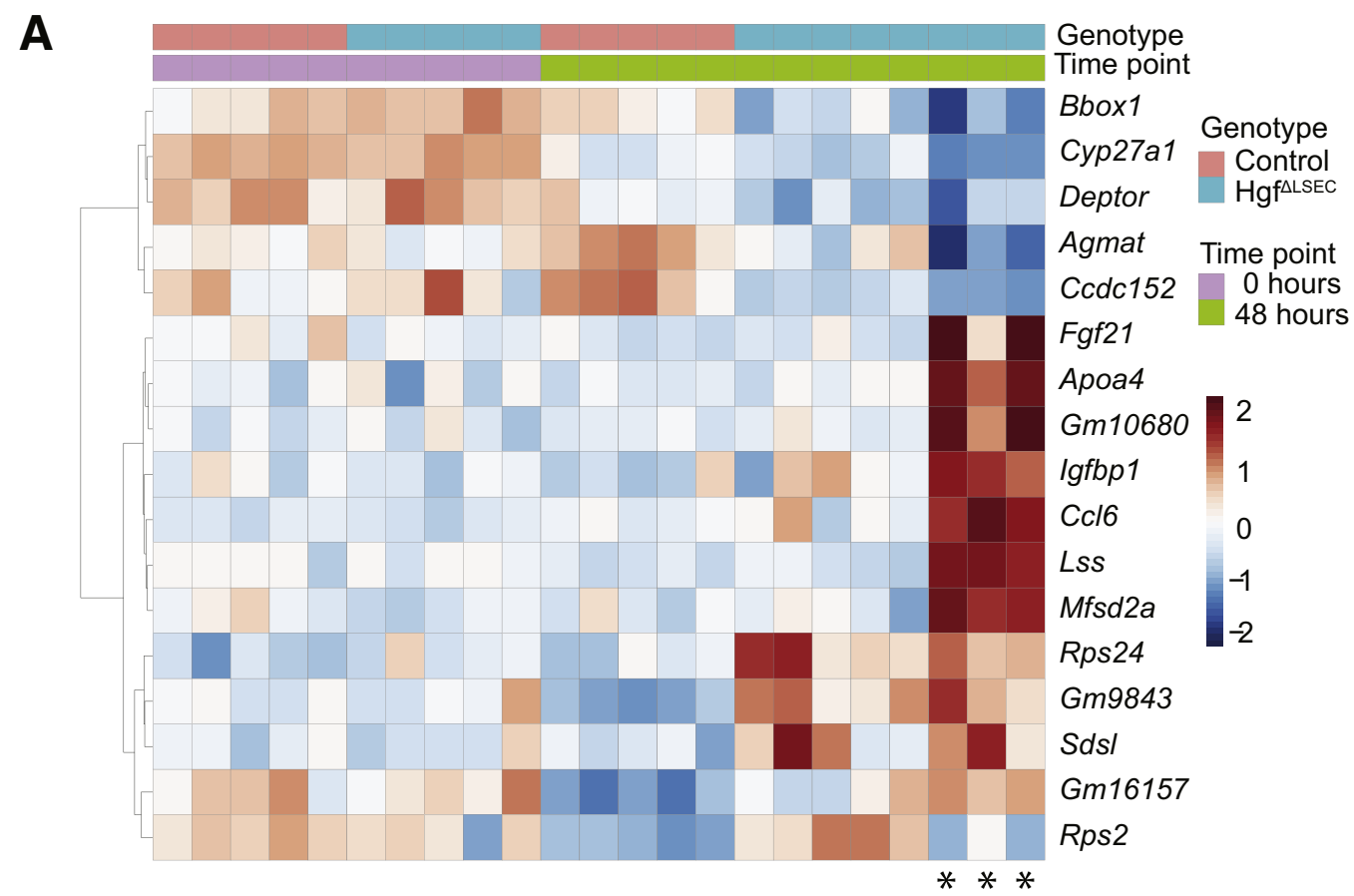

B
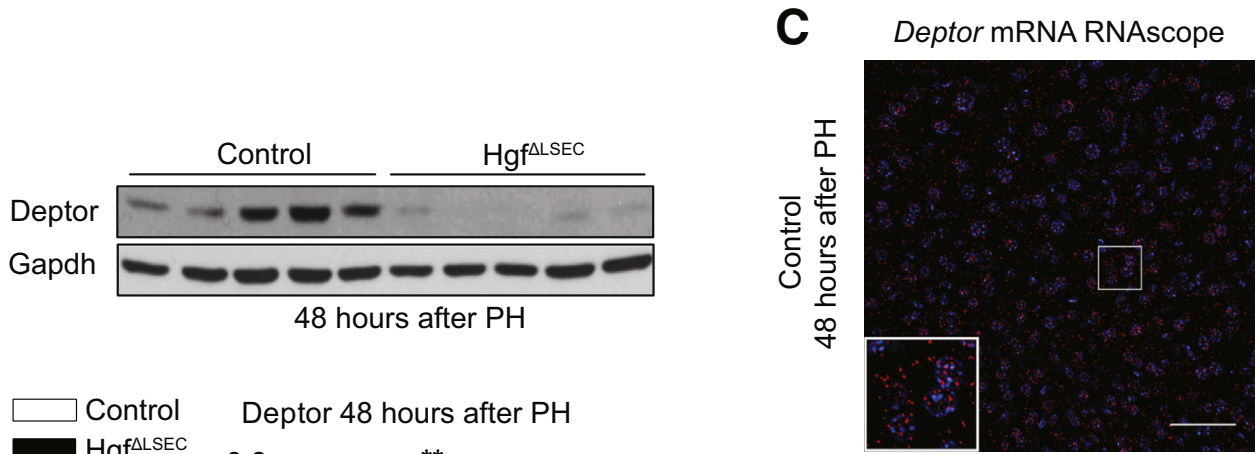

48 hours after $\mathrm{PH}$

Control Deptor 48 hours after $\mathrm{PH}$
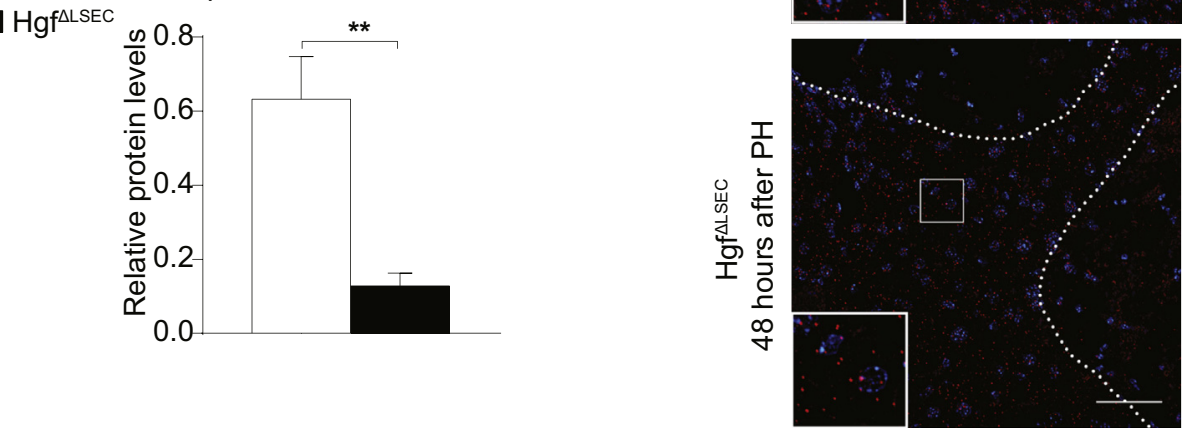

Figure 8 Gene expression analysis identifies down-regulation of Deptor on partial hepatectomy (PH) of Hgf ${ }^{\Delta L S E C}$ livers. A: Heat map of RNA sequencing

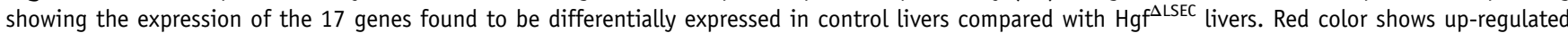
genes, and blue color shows down-regulated genes. The color scale represents the z-scaled gene expression levels. Hgf ${ }^{\Delta L E C}$ livers with necrosis are marked asterisks. B: Down-regulation of hepatic Deptor 48 hours after $70 \% \mathrm{PH}$ in $\mathrm{Hgf}^{\Delta \mathrm{LSEC}}$ compared with control mice. C: Fluorescence in situ hybridization of Deptor on liver sections of control and $\mathrm{Hgf}^{\triangle L S E C}$ mice. Dotted white lines indicate necrotic area. Boxed areas are shown at higher magnification in the insets. Data are expressed as means \pm SEM (B). $n \geq 5$ (A); $n=5$ (B); $n=4$ (C). ${ }^{*} P<0.01$. Scale bar $=50 \mu \mathrm{m}$ (C). Original magnification, $\times 60$ (insets). Gapdh, glyceraldehyde-3-phosphate dehydrogenase.

signaling leads to fewer offspring, reduced body weight, decreased liver/body weight ratio, and effaced hepatic zonation as well as lower plasma cholesterol levels. ${ }^{17}$ Similarly, developmental or metabolic changes have not been described in tamoxifen-inducible deletion of $\mathrm{Hgf}$ in vascular endothelial-cadherin-positive ECs. ${ }^{27}$ To study angiocrine Hgf signaling during embryonic development and adult homeostasis, a novel mouse model was generated to delete $H g f$ in LSECs during early embryonic development. Thereby, it was shown that angiocrine Hgf signaling 
does not affect embryonic liver development as well as adult liver zonation and structure under steady-state conditions but leads to reduced total body, liver, and spleen weights. Although Stab2-Cre activity is predominantly found in sinusoidal ECs, some reporter activity has been described, especially in larger vessels at E12.5, ${ }^{28}$ so that we cannot exclude that altered angiocrine $\mathrm{Hgf}$ signaling in nonsinusoidal ECs may contribute to the small decrease in total body weight. Livers of global $\mathrm{Hgf}$ knockout mice are known to be severely reduced in size from E12.5 and more pronounced at E14.5 and die subsequently. ${ }^{9}$ In this context, $\mathrm{Hgf}$ derived from other hepatic cells may compensate for lack of angiocrine $\mathrm{Hgf}$ signaling in $\mathrm{Hgf}^{\triangle \mathrm{LSEC}}$. Because the liver contributes to a multitude of metabolic functions throughout the body, angiocrine Hgf may control body growth via global metabolism. ${ }^{41}$ Although certain signaling pathways involving insulin-like growth factor (Igf), Hippo, or pumilio and FBF (PUF) family proteins have been described to control body and organ size, the mechanisms that determine global growth and size are still largely unknown. ${ }^{42-44}$ Although these underlying mechanisms are not well characterized, total body weight and liver weight are in general tightly related, as indicated by a constant liver/body weight ratio. ${ }^{45}$ Therefore, the observed reduction of body and liver weight with a constant liver/body weight ratio in $\mathrm{Hgf}^{\Delta \mathrm{LSEC}}$ demonstrates that this interrelation is preserved in these mice. This is in contrast to mice with defective angiocrine Wnt signaling that show a decreased total body weight as well as a lower liver/body weight ratio due to a decreased population of Axin2 $^{+}$pericentral hepatocytes. ${ }^{17,40}$ Our findings indicate that angiocrine $\mathrm{Hgf}$ controls body and liver size by other means, potentially by modulation of global growth and metabolism. Therefore, except for lower total body and liver weights, $\mathrm{Hgf}^{\mathrm{ALSEC}}$ did not show major alterations of liver development and function or general impairment.

The liver is the only solid organ in mammals that can regenerate after removal of a significant amount of tissue. ${ }^{30}$ Intriguingly, $\mathrm{Hgf}^{\Delta \mathrm{LSEC}}$ mice showed impaired dynamics of liver regeneration, enhanced hepatic necrosis, and a reduced liver/body weight ratio at 72 hours after $\mathrm{PH}$, despite the fact that both hepatic stellate cells and LSECs contribute to the increase of Hgf production after $\mathrm{PH}^{34,41}$ Therefore, stellate cells and Kupffer cells cannot compensate for the lack of endothelial $\mathrm{Hgf}$ in this context. Besides Hgf secretion by other cells, Hgf stored in the liver extracellular matrix ${ }^{39}$ may also be a source compensating for deficient angiocrine $\mathrm{Hgf}$ in $\mathrm{Hgf}^{\triangle \mathrm{LSEC}}$. Hepatocyte proliferation analysis indicated that the early phase of regeneration is disturbed in $\mathrm{Hgf}^{\triangle \mathrm{LSEC}}$. In comparison to hepatocyte-specific c-Met knockout mice, $\mathrm{Hgf}^{\Delta \mathrm{LSEC}}$ showed a less marked reduction of proliferation, indicating residual activity of the $\mathrm{Hgf} / \mathrm{c}-\mathrm{Met}$ axis. ${ }^{36}$ Although this residual $\mathrm{Hgf} / \mathrm{c}-\mathrm{Met}$ activity was not sufficient to prevent tissue necrosis in $\mathrm{Hgf}^{\triangle \mathrm{LSEC}}$, humane end points were only reached in $12.8 \%$ of mutant mice in our study, whereas a lethality of $80 \%$ is seen in hepatocyte-specific $c$-Met knockout after $\mathrm{PH} .{ }^{26}$ Therefore, decreased c-Met activation is the probable cause of the increased necrotic tissue damage and lethality observed in $\mathrm{Hgf}^{\triangle \mathrm{LSEC}}$.

As the mice that did not reach humane end points showed a normal liver/body weight ratio in the late phases of regeneration, lack of angiocrine $\mathrm{Hgf}$ in $\mathrm{Hgf}^{\Delta \mathrm{LSEC}}$ appears to be compensated by other sources of $\mathrm{Hgf}$ or other factors known to be involved in liver regeneration, such as Il6, epidermal growth factor family, fibroblast growth factors, vascular endothelial growth factor, and Igfs. ${ }^{46}$ To characterize the molecular events during regeneration in $\mathrm{Hgf}^{\Delta \mathrm{LSEC}}$, RNA sequencing was performed. Herein, Igfbpl was strongly up-regulated in $\mathrm{Hgf}^{\Delta \mathrm{LSEC}}$ livers 48 hours after $\mathrm{PH}$. insulin-like growth factor-binding protein 1 (Igfbp1) protein can specifically bind and modulate the bioavailability of insulin-like growth factors (Igf-I and Igf-II), which are involved in liver regeneration. Igfbpl knockout mice demonstrate impaired liver regeneration after $\mathrm{PH}$, characterized by liver necrosis and reduced and delayed hepatocyte regeneration. ${ }^{47}$ Therefore, up-regulation of Igfbpl in $\mathrm{Hgf}^{\Delta \mathrm{LSEC}}$ livers could be one compensative response to stimulate liver regeneration after $\mathrm{PH}$.

On the other hand, Deptor was identified as one of the genes most strongly down-regulated in $\mathrm{Hgf}^{\Delta \mathrm{LSEC}}$ livers 48 hours after $\mathrm{PH}$. Deptor is an inhibitor of mammalian target of rapamycin (mTOR), a serine/threonine kinase, known to regulate mRNA translation, autophagy, and cell survival. ${ }^{48-51}$ Loss of Deptor was shown to induce apoptosis through down-regulation of phosphatidylinositol 3-kinase/Akt signaling. ${ }^{52}$ Downregulation of Deptor could, therefore, contribute to decreased hepatocyte proliferation in $\mathrm{Hgf}^{\triangle \mathrm{LSEC}}$. Our data indicate that reduced expression of Deptor is functionally involved in the reduced proliferation of hepatocytes at 48 hours after $\mathrm{PH}$ in $\mathrm{Hgf}^{\Delta \mathrm{LSEC}}$. As direct regulation of Deptor expression by $\mathrm{Hgf}$ stimulation of AML-12 cells was not observed, it is likely that either angiocrine $\mathrm{Hgf} / \mathrm{c}-\mathrm{Met}$ activation may act synergistically and nonredundantly with other angiocrine or paracrine signals in this context or Deptor induction occurs via secondary mediators secreted by other nonparenchymal cells in response to angiocrine Hgf. Further studies are required to assess whether there is a direct link between Hgf and Deptor in vivo and whether this regulation is context dependent.

In summary, we generated, for the first time, a novel mouse model lacking $\mathrm{Hgf}$ in the liver sinusoidal endothelial cells already from embryonic development onwards. These mice were not only smaller in body, liver, and spleen weight, but also revealed liver damage and impeded liver regeneration after partial hepatectomy. Functionally, reduced hepatocyte proliferation in $\mathrm{Hgf}^{\Delta \mathrm{LSEC}}$ could be related to decreased induction of Deptor, a regulator of proliferation in hepatocytes. Angiocrine Hgf signaling is not only essential for liver regeneration and preventing excessive organ damage, but also for the control of body and organ growth. 


\section{Acknowledgments}

We thank Jochen Weber and Hiltrud Schönhaber for excellent technical support; and Ana Hidalgo-Sastre, Suyang Zhong, Yang Wang, and Carolin Mogler for discussion and comments during research.

\section{Author Contributions}

C.G., G.v.F., and D.H. conceived and designed the study; X.-j.Z. and V.O. performed experiments and analyzed the data; T.E. and R.O. performed RNA sequencing and analyzed the data; all authors wrote and reviewed the manuscript and approved it before submission.

\section{Supplemental Data}

Supplemental material for this article can be found at https://doi.org/10.1016/j.ajpath.2019.10.009.

\section{References}

1. Nakamura T, Nawa K, Ichihara A: Partial purification and characterization of hepatocyte growth factor from serum of hepatectomized rats. Biochem Biophys Res Commun 1984, 122:1450-1459

2. Russell WE, McGowan JA, Bucher NL: Partial characterization of a hepatocyte growth factor from rat platelets. J Cell Physiol 1984, 119: $183-192$

3. Fajardo-Puerta AB, Mato Prado M, Frampton AE, Jiao LR: Gene of the month: HGF. J Clin Pathol 2016, 69:575-579

4. Lindroos PM, Zarnegar R, Michalopoulos GK: Hepatocyte growth factor (hepatopoietin A) rapidly increases in plasma before DNA synthesis and liver regeneration stimulated by partial hepatectomy and carbon tetrachloride administration. Hepatology 1991, 13: $743-750$

5. Migliore C, Giordano S: Molecular cancer therapy: can our expectation be MET? Eur J Cancer 2008, 44:641-651

6. Giordano S, Columbano A: Met as a therapeutic target in HCC: facts and hopes. J Hepatol 2014, 60:442-452

7. Matsumoto K, Nakamura T: Emerging multipotent aspects of hepatocyte growth factor. J Biochem 1996, 119:591-600

8. Bladt F, Riethmacher D, Isenmann S, Aguzzi A, Birchmeier C: Essential role for the c-met receptor in the migration of myogenic precursor cells into the limb bud. Nature 1995, 376:768-771

9. Schmidt C, Bladt F, Goedecke S, Brinkmann V, Zschiesche W, Sharpe M, Gherardi E, Birchmeier C: Scatter factor/hepatocyte growth factor is essential for liver development. Nature 1995, 373: 699-702

10. Nakamura T, Mizuno S: The discovery of hepatocyte growth factor (HGF) and its significance for cell biology, life sciences and clinical medicine. Proc Jpn Acad Ser B Phys Biol Sci 2010, 86:588-610

11. Maher JJ: Cell-specific expression of hepatocyte growth factor in liver: upregulation in sinusoidal endothelial cells after carbon tetrachloride. J Clin Invest 1993, 91:2244-2252

12. Noji S, Tashiro K, Koyama E, Nohno T, Ohyama K, Taniguchi S, Nakamura T: Expression of hepatocyte growth factor gene in endothelial and Kupffer cells of damaged rat livers, as revealed by in situ hybridization. Biochem Biophys Res Commun 1990, 173:42-47

13. Hu J, Srivastava K, Wieland M, Runge A, Mogler C, Besemfelder E, Terhardt D, Vogel MJ, Cao L, Korn C, Bartels S, Thomas M,
Augustin HG: Endothelial cell-derived angiopoietin-2 controls liver regeneration as a spatiotemporal rheostat. Science 2014, 343:416-419

14. Ding BS, Nolan DJ, Butler JM, James D, Babazadeh AO, Rosenwaks Z, Mittal V, Kobayashi H, Shido K, Lyden D, Sato TN, Rabbany SY, Rafii S: Inductive angiocrine signals from sinusoidal endothelium are required for liver regeneration. Nature 2010, 468: 310-315

15. LeCouter J, Moritz DR, Li B, Phillips GL, Liang XH, Gerber HP, Hillan KJ, Ferrara N: Angiogenesis-independent endothelial protection of liver: role of VEGFR-1. Science 2003, 299:890-893

16. Koch PS, Olsavszky V, Ulbrich F, Sticht C, Demory A, Leibing T, Henzler T, Meyer M, Zierow J, Schneider S, Breitkopf-Heinlein K, Gaitantzi H, Spencer-Dene B, Arnold B, Klapproth K, Schledzewski K, Goerdt S, Geraud C: Angiocrine Bmp2 signaling in murine liver controls normal iron homeostasis. Blood 2017, 129: 415-419

17. Leibing T, Geraud C, Augustin I, Boutros M, Augustin HG, Okun JG, Langhans CD, Zierow J, Wohlfeil SA, Olsavszky V, Schledzewski K, Goerdt S, Koch PS: Angiocrine Wnt signaling controls liver growth and metabolic maturation in mice. Hepatology 2018, 68:707-722

18. Rafii S, Butler JM, Ding BS: Angiocrine functions of organ-specific endothelial cells. Nature 2016, 529:316-325

19. Ding BS, Cao Z, Lis R, Nolan DJ, Guo P, Simons M, Penfold ME, Shido K, Rabbany SY, Rafii S: Divergent angiocrine signals from vascular niche balance liver regeneration and fibrosis. Nature 2014, 505:97-102

20. Kenessey I, Keszthelyi M, Kramer Z, Berta J, Adam A, Dobos J, Mildner M, Flachner B, Cseh S, Barna G, Szokol B, Orfi L, Keri G, Dome B, Klepetko W, Timar J, Tovari J: Inhibition of c-Met with the specific small molecule tyrosine kinase inhibitor SU11274 decreases growth and metastasis formation of experimental human melanoma. Curr Cancer Drug Targets 2010, 10:332-342

21. Sakata H, Takayama H, Sharp R, Rubin JS, Merlino G, LaRochelle WJ: Hepatocyte growth factor/scatter factor overexpression induces growth, abnormal development, and tumor formation in transgenic mouse livers. Cell Growth Differ 1996, 7: $1513-1523$

22. Muratsu J, Iwabayashi M, Sanada F, Taniyama Y, Otsu R, Rakugi H, Morishita R: Hepatocyte growth factor prevented high-fat dietinduced obesity and improved insulin resistance in mice. Sci Rep 2017, 7:130

23. Zarnegar $\mathrm{R}$, DeFrances $\mathrm{MC}$, Kost DP, Lindroos $\mathrm{P}$, Michalopoulos GK: Expression of hepatocyte growth factor mRNA in regenerating rat liver after partial hepatectomy. Biochem Biophys Res Commun 1991, 177:559-565

24. Yanagita K, Nagaike M, Ishibashi H, Niho Y, Matsumoto K Nakamura T: Lung may have an endocrine function producing hepatocyte growth-factor in response to injury of distal organs. Biochem Biophys Res Commun 1992, 182:802-809

25. Kono S, Nagaike M, Matsumoto K, Nakamura T: Marked induction of hepatocyte growth factor mRNA in intact kidney and spleen in response to injury of distant organs. Biochem Biophys Res Commun 1992, 186:991-998

26. Huh CG, Factor VM, Sanchez A, Uchida K, Conner EA, Thorgeirsson SS: Hepatocyte growth factor/c-met signaling pathway is required for efficient liver regeneration and repair. Proc Natl Acad Sci U S A 2004, 101:4477-4482

27. Cao Z, Ye T, Sun Y, Ji G, Shido K, Chen Y, Luo L, Na F, Li X, Huang Z, Ko JL, Mittal V, Qiao L, Chen C, Martinez FJ, Rafii S, Ding BS: Targeting the vascular and perivascular niches as a regenerative therapy for lung and liver fibrosis. Sci Transl Med 2017, 9:eaai8710

28. Geraud C, Koch PS, Zierow J, Klapproth K, Busch K, Olsavszky V Leibing T, Demory A, Ulbrich F, Diett M, Singh S, Sticht C, Breitkopf-Heinlein K, Richter K, Karppinen SM, Pihlajaniemi T, Arnold B, Rodewald HR, Augustin HG, Schledzewski K, Goerdt S: 
GATA4-dependent organ-specific endothelial differentiation controls liver development and embryonic hematopoiesis. J Clin Invest 2017, 127:1099-1114

29. Phaneuf D, Moscioni AD, LeClair C, Raper SE, Wilson JM: Generation of a mouse expressing a conditional knockout of the hepatocyte growth factor gene: demonstration of impaired liver regeneration. DNA Cell Biol 2004, 23:592-603

30. Mitchell C, Willenbring $\mathrm{H}$ : A reproducible and well-tolerated method for $2 / 3$ partial hepatectomy in mice. Nat Protoc 2008, 3:1167-1170

31. Bankhead P, Loughrey MB, Fernandez JA, Dombrowski Y, McArt DG, Dunne PD, McQuaid S, Gray RT, Murray LJ, Coleman HG, James JA, Salto-Tellez M, Hamilton PW: QuPath: open source software for digital pathology image analysis. Sci Rep 2017, 7:16878

32. Love MI, Huber W, Anders S: Moderated estimation of fold change and dispersion for RNA-seq data with DESeq2. Genome Biol 2014, $15: 550$

33. Miyaoka Y, Ebato K, Kato H, Arakawa S, Shimizu S, Miyajima A: Hypertrophy and unconventional cell division of hepatocytes underlie liver regeneration. Curr Biol 2012, 22:1166-1175

34. Michalopoulos GK: Liver regeneration after partial hepatectomy: critical analysis of mechanistic dilemmas. Am J Pathol 2010, 176:2-13

35. Uehara Y, Minowa O, Mori C, Shiota K, Kuno J, Noda T, Kitamura N: Placental defect and embryonic lethality in mice lacking hepatocyte growth factor/scatter factor. Nature 1995, 373:702-705

36. Borowiak M, Garratt AN, Wustefeld T, Strehle M, Trautwein C, Birchmeier C: Met provides essential signals for liver regeneration. Proc Natl Acad Sci U S A 2004, 101:10608-10613

37. Paranjpe S, Bowen WC, Bell AW, Nejak-Bowen K, Luo JH, Michalopoulos GK: Cell cycle effects resulting from inhibition of hepatocyte growth factor and its receptor c-Met in regenerating rat livers by RNA interference. Hepatology 2007, 45:1471-1477

38. Ishikawa T, Factor VM, Marquardt JU, Raggi C, Seo D, Kitade M, Conner EA, Thorgeirsson SS: Hepatocyte growth factor/c-met signaling is required for stem-cell-mediated liver regeneration in mice. Hepatology 2012, 55:1215-1226

39. Nejak-Bowen K, Orr A, Bowen WC Jr, Michalopoulos GK: Conditional genetic elimination of hepatocyte growth factor in mice compromises liver regeneration after partial hepatectomy. PLoS One 2013, 8:e59836

40. Wang B, Zhao L, Fish M, Logan CY, Nusse R: Self-renewing diploid Axin2(+) cells fuel homeostatic renewal of the liver. Nature 2015, 524:180-185
41. Michalopoulos GK: Principles of liver regeneration and growth homeostasis. Compr Physiol 2013, 3:485-513

42. Lin K, Qiang W, Zhu M, Ding Y, Shi Q, Chen X, Zsiros E, Wang K, Yang X, Kurita T, Xu EY: Mammalian Pum1 and Pum2 control body size via translational regulation of the cell cycle inhibitor Cdkn1b. Cell Rep 2019, 26:2434-2450.e6

43. Stanger BZ: Organ size determination and the limits of regulation. Cell Cycle 2008, 7:318-324

44. Yu FX, Zhao B, Guan KL: Hippo pathway in organ size control, tissue homeostasis, and cancer. Cell 2015, 163:811-828

45. Lehmann K, Tschuor C, Rickenbacher A, Jang JH, Oberkofler CE, Tschopp O, Schultze SM, Raptis DA, Weber A, Graf R, Humar B, Clavien PA: Liver failure after extended hepatectomy in mice is mediated by a p21-dependent barrier to liver regeneration. Gastroenterology 2012, 143:1609-1619.e4

46. Bohm F, Kohler UA, Speicher T, Werner S: Regulation of liver regeneration by growth factors and cytokines. EMBO Mol Med 2010, 2:294-305

47. Leu JI, Crissey MA, Craig LE, Taub R: Impaired hepatocyte DNA synthetic response posthepatectomy in insulin-like growth factor binding protein 1-deficient mice with defects in C/EBP beta and mitogen-activated protein kinase/extracellular signal-regulated kinase regulation. Mol Cell Biol 2003, 23:1251-1259

48. Catena V, Fanciulli M: Deptor: not only a mTOR inhibitor. J Exp Clin Cancer Res 2017, 36:12

49. Peterson TR, Laplante M, Thoreen CC, Sancak Y, Kang SA, Kuehl WM, Gray NS, Sabatini DM: DEPTOR is an mTOR inhibitor frequently overexpressed in multiple myeloma cells and required for their survival. Cell 2009, 137:873-886

50. Zhang H, Chen J, Zeng Z, Que W, Zhou L: Knockdown of DEPTOR induces apoptosis, increases chemosensitivity to doxorubicin and suppresses autophagy in RPMI-8226 human multiple myeloma cells in vitro. Int J Mol Med 2013, 31:1127-1134

51. Zhang HR, Chen JM, Zeng ZY, Que WZ: Knockdown of DEPTOR inhibits cell proliferation and increases chemosensitivity to melphalan in human multiple myeloma RPMI-8226 cells via inhibiting PI3K/AKT activity. J Int Med Res 2013, 41:584-595

52. Srinivas KP, Viji R, Dan VM, Sajitha IS, Prakash R, Rahul PV, Santhoshkumar TR, Lakshmi S, Pillai MR: DEPTOR promotes survival of cervical squamous cell carcinoma cells and its silencing induces apoptosis through downregulating PI3K/AKT and by up-regulating p38 MAP kinase. Oncotarget 2016, 7:24154-24171 